\title{
Probabilistic Model-Based Background Subtraction
}

\author{
Volker Krüger $^{1}$, Jakob Anderson ${ }^{2}$, and Thomas Prehn ${ }^{2}$ \\ 1 Aalborg Media Lab, Aalborg University, \\ Copenhagen, Lautrupvang 15, 2750 Ballerup \\ 2 Aalborg University Esbjerg, \\ Niels Bohrs Vej 8, 6700 Esbjerg, Denmark
}

\begin{abstract}
Usually, background subtraction is approached as a pixelbased process, and the output is (a possibly thresholded) image where each pixel reflects, independent from its neighboring pixels, the likelihood of itself belonging to a foreground object. What is neglected for better output is the correlation between pixels. In this paper we introduce a model-based background subtraction approach which facilitates prior knowledge of pixel correlations for clearer and better results. Model knowledge is being learned from good training video data, the data is stored for fast access in a hierarchical manner. Bayesian propagation over time is used for proper model selection and tracking during model-based background subtraction. Bayes propagation is attractive in our application as it allows to deal with uncertainties during tracking. We have tested our approach on suitable outdoor video data.
\end{abstract}

\section{Introduction}

Companies and scientists work on vision systems that are expected to work in real-world scenarios. Car companies work, e.g., on road sign and pedestrian detection and due to the threat of terrorism, biometric vision systems and surveillance applications are under development.

All these approaches work well in controlled environments, e.g., attempts to recognize humans by their face and gait has proven to be very successful in a lab environment. However, in uncontrolled environments, such as outdoor scenarios, the approaches disgracefully fail, e.g., the gait recognition drops from $99 \%$ to merely $30 \%$. This is mainly due to low quality video data, the often small number of pixels on target and visual distractors such as shadows and strong illumination variations.

What is needed are special feature extraction techniques that are robust to outdoor distortions and that can cope with low-quality video data. One of the most common feature extraction techniques in surveillance applications is background subtraction (BGS) [5, 3, 9, 6. BGS approaches assume a stable camera. They are able to learn a background as well as possible local image variations of it, thus generating a background model even of non-rigid background objects. 
During application the model is compared with novel video images and pixels are marked according to the belief that they are fitting the background model. Generally, BGS methods have the following drawbacks:

1. BGS techniques are able to detect "interesting" images areas, i.e., image areas that are sufficiently different from the learned background model. Thus, BGS approaches are able to detect, e.g., a person and the shadow that he/she throws. However, BGS approaches are not able to distinguish between a foreground object and its shadow.

2. Very often, the same objects causes a different output when the scenario changes: E.g. the BGS output for a person walking on green grass or gray concrete may be different.

In this paper we present a Model-based Background Subtracting (MBGS) method that learns not only a background model but also a foreground model. The "classical" background subtraction detects the region of interests while the foreground models are being applied to the classical BGS output to "clean up" possible noise. To reach a maximum of robustness, we apply statistical propagation techniques to the likelihood measures of the BGS.

Having the recent gait recognition attempts in mind, we have applied the following limitations to our discussion (however, the methodology is general enough that we do not see any limit of generality in the chosen setup):

1. We consider only humans as objects and ignore objects that look different from humans.

2. We limit our discussion to silhouettes of humans as they deliver a fairly clothing-independent description of an individual.

The Model-based Background Subtraction System (MBGS System) consists of a learning part to learn possible foreground objects and a MBGS part, where the output of a classical BGS is verified using the previously trained foreground object knowledge.

To learn and represent the foreground knowledge (here silhouettes of humans) is non-trivial due to the absence of a suitable vector space. One possibility is to describe the data in a hierarchical manner, using a suitable metric and a suitable representation of dynamics between the silhouettes. Since we use the silhouettes as density functions where each pixel describes the likelihood of being either foreground or background, we use the Kullback-Leibler distance to compare the silhouettes, k-means clustering is used for clustering similar ones. Similar approaches for hierarchical contour representation can be found in [4,14.

In the second part, we again consider the contours as densities over spatial coordinates and use normalized correlation to compute the similarity between the silhouette density and the computed one in the input image. Tracking and silhouette selection is being done using Bayesian propagation over time. It can be applied directly since we are dealing with densities and it has the advantage that it considers the uncertainty in the estimation of the tracking parameters and the silhouette selection. The densities in the Bayesian propagation are approximated 
using an enhancement of the well-known Condensation method [7. A similar enhancement of the Condensation method has been applied in video based face recognition 12 .

The remainder of this paper is organized as follows: In Sec. 2 we introduce the learning approaches. The actual BGS method is discussed in Sec. 3. We conclude with experimental results in Sec. 4 and final remarks are in Sec. 5 .

\section{$2 \quad$ Learning and Representation of Foreground Objects}

In order to make use of foreground model knowledge, the MBGS system needs to be able to:

- learn a model representation for possibly a number of different and non-rigid objects from video data and

- quickly access the proper model information during application of the MBGS.

Our main idea is the following: Apply the classical BGS to a scenario that is controlled in a manner that facilitates the learning process. In our case, since we want to learn silhouettes of humans, that only humans are visible in the scene during training and that the background variations are kept as small as possible to minimize distortions. Then, use this video data to learn the proper model knowledge.

After the application of a classical BGS, applying mean-shift tracking [1] allows to extract from the BGS output-data a sequence of small image patches containing, centered, the silhouette. This procedure is the same as the one presented in [10, however, with the difference that here we do not threshold the BGS output but use probabilistic silhouettes (instead of binary ones as in [10]). Thus the silhouettes still contain for each silhouette pixel the belief of being a foreground pixel.

To organize this data we use, similar to 4, a combination of tree structuring and k-means clustering. We use a top down approach: The first level is the root of the hierarchy which contains all the exemplars. Then the second level is constructed by using a the k-means clustering to cluster the exemplars from the root. The third level is constructed by clustering each cluster from the second level, again, using k-means, see Fig. 1 for an example. The k-means clustering uses the Kullback-Leibler divergence measure which measures the similarity between two density functions $p$ and $q$ :

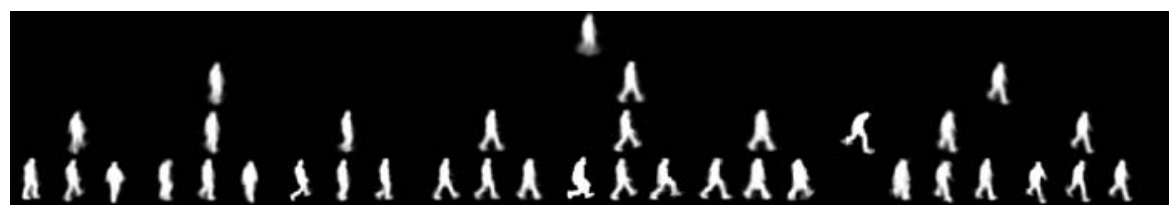

Fig. 1. An example of our clustering approach: 30 exemplars with $\mathrm{K}=3$ and the algorithm stops after reaching 3 levels 

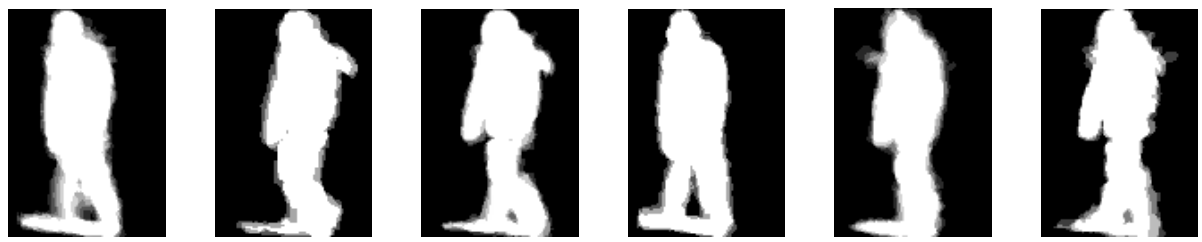

Fig. 2. The five images show the cluster centers computed from a training sequence of a single individual

$$
K \operatorname{LDist}(p, q)=\int p(\mathbf{x}) \log \frac{p(\mathbf{x})}{q(\mathbf{x})} d \mathbf{x} .
$$

$\operatorname{KLDist}(p, q)$ is non-negative and only zero if $p$ and $q$ coincide.

See Fig. 2 for an example clustering of a training sequence of a single individual (as training data, we chose here non-optimal data for better visualization). The tree structure facilitates a fast search of exemplars along the tree vertices, and the cluster centers are either used to apply MBGS on a coarse level or they are used as proto-types, as in [4, to direct the search to a finer level in the hierarchy. Once the tree is constructed, we generate a Markov transition matrix: Assuming that the change over time from one silhouette to a next one can be understood as a first order Markov process, the Markov transition matrix $M_{i j}^{l}$ describes the transition probability of silhouette $s_{j}$ following after silhouette $s_{i}$ at level $l$ in the hierarchy. During MBGS application particle filtering [8, 11, 2] will be used to find the proper silhouette (see Sec. 3). The propagation of silhouettes over time is non-trivial, as silhouette do not form a vector space. However, what is sufficient is a (not necessarily symmetric) metric space, i.e., given a silhouette $s_{i}$, all silhouettes are needed that are close according to a given (not necessarily symmetric) metric. In the tree structure similar contours are clustered which facilitates the propagation process. The Markov transition matrix $M_{i j}$ on the other hand describes directly the transition likelihoods between clusters.

\section{$3 \quad$ Applying Background Subtraction and Recognizing Foreground Objects}

The MBGS system is built as an extension to a pixel based BGS approach. It uses foreground models to define likely correlations between neighbored pixels in the output $P(\mathbf{x})$ of the BGS application.

Each pixel in the image $P(\mathbf{x})$ contains a value in the range $[0,1]$, where 1 indicates the highest probability of a pixel being a foreground pixel. A model in the hierarchy can be chosen and deformed according to a 4 -D vector

$$
\theta=[i, s, x, y]
$$

where $x$ and $y$ denote the position of the silhouette in the image $P, s$ its scale, and $i$ is a natural number that refers to a silhouette in the hierarchy. 
Presently, the "matching" is done by normalized correlation between a model silhouette density, parameterized according to a deformation vector $\theta_{t}$ and the appropriate region of interest in the BGS image $P_{t}(\mathbf{x})$, appropriately normalized.

In order to find at each time-step $t$ the most likely $\theta_{t}$ in the image $P_{t}(\mathbf{x})$, we use Bayesian propagation over time

$$
\begin{aligned}
& p\left(\theta_{t} \mid P_{1}, P_{2}, \ldots, P_{t}\right) \equiv p_{t}\left(\alpha_{t}, i_{t}\right) \\
& =\sum_{i_{t-1}} \int_{\alpha_{t-1}} p\left(P_{t} \mid \alpha_{t}, i_{t}\right) \\
& \quad p\left(\alpha_{t}, i_{t} \mid \alpha_{t-1}, i_{t-1}\right) p_{t-1}\left(\alpha_{t-1}, i_{t-1}\right)
\end{aligned}
$$

with $\alpha_{t}=[s, x, y]_{t} ; P_{t}$ denotes the probability images while $p$ denotes density functions. In order to approximate the posteriori density $p\left(\theta_{t} \mid P_{1}, P_{2}, \ldots, P_{t}\right)$ we use sequential importance sampling (SIS) 2, 7, 11, 13.

Bayesian propagation over time allows us to take into account the uncertainty in the estimated parameters. The uncertainty can be made explicit by computing the entropy of $p$.

Monte Carlo methods, like the SIS, use random samples for the approximation of a density function.

Our MBGS system uses separate sample sets for each object in the input image. A new sample set is constructed every time a new object in the video image matches sufficiently well any of the exemplars in the exemplar database.

As the diffusion density $p\left(\alpha_{t}, i_{t} \mid \alpha_{t-1}, i_{t-1}\right)$ in Eq. 3 we use the Brownian motion model due to the absence of a better one. For the propagation of the position and scale parameters, $x, y$, and $s$, this is straight forward. For the detection and the propagation of the silhouette we use the following strategy: The likelihood for selecting a silhouette from a certain silhouette cluster in the hierarchy is computed from the Markov transition matrix $M$ by marginalizing over the silhouettes in that particular cluster. Within a cluster, the new silhouette is then chosen randomly. The reason for this is that since our training data is too little so that the Markov transition matrix $M$ appeared to be specific to the training videos.

The basic strategy to find the proper silhouette is similar to [12] where the authors find the right identity of a person in a video. In [12, the identity does not change, and the fact that all the particles in the SIS particle filter converge to the same identity is wanted. However, in our case, once all particles have converged to one silhouette, the system would never be able to change anymore to a different silhouette. The diffusion strategy has to assure that enough particles converge to the correct silhouette while at the same time they have the chance to converge to a different one when the shape in the input image changes.

\section{Experiments}

In this section we present qualitative and quantitative results obtained from experiments with our MBGS implementation. The experiments clearly show the 
potential of an effective MBGS approach. The purpose of the experiments was to verify that the drawbacks of the classical BGS approach, which were mentioned in section 1. can be remedied with MBGS. More specifically the MBGS system verifies the following:

1. Because shadows are not part of the model information provided, these will be classified as background by the implemented MBGS approach. In fact, most non-model object types will be classified as background, and therefore MBGS allows for effective object type filtering.

2. The output presented from the MBGS does not vary, even when the scenario changes significantly. If a model is presented as output, it is always presented intact. The object behind a silhouette is therefore always determinable.

Qualitative verification is done by comparing our MBGS system (top right in the Figs (36) with two previously developed pixel-based BGS approaches: One is the non-parametric approach developed at Maryland (UMD BGS, bottom left in the Figs.) [3. The other BGS, which utilizes a recursive image noise filtering technique, has been developed along with this work.

Figure 3 shows a scenario, with a pedestrian walking behind trees, thereby at times being occluded. The output of two pixel-based approaches is shown in the lower part of the figure. Notice that the shadow cast by the pedestrian is

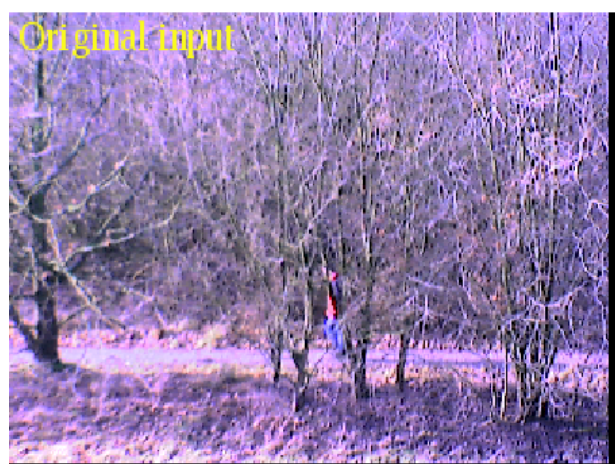

UMD BGS

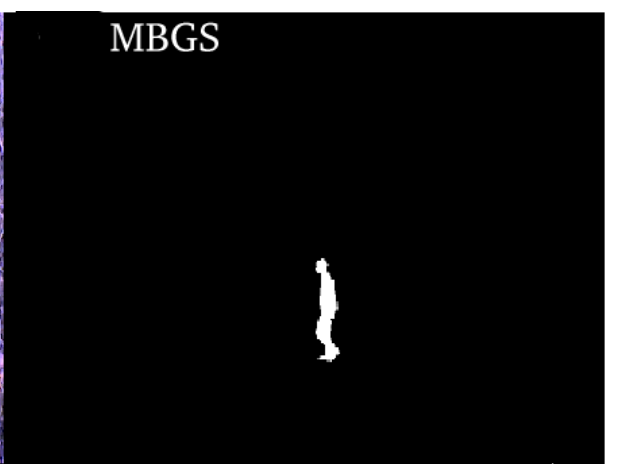

BGS

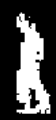

Fig. 3. BGS approach comparison of cases of heavy shadow 


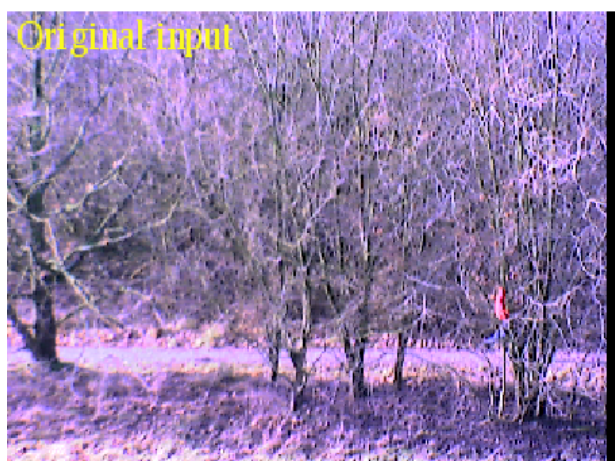

UMD BGS

\section{MBGS}

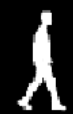

\section{BGS}

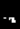

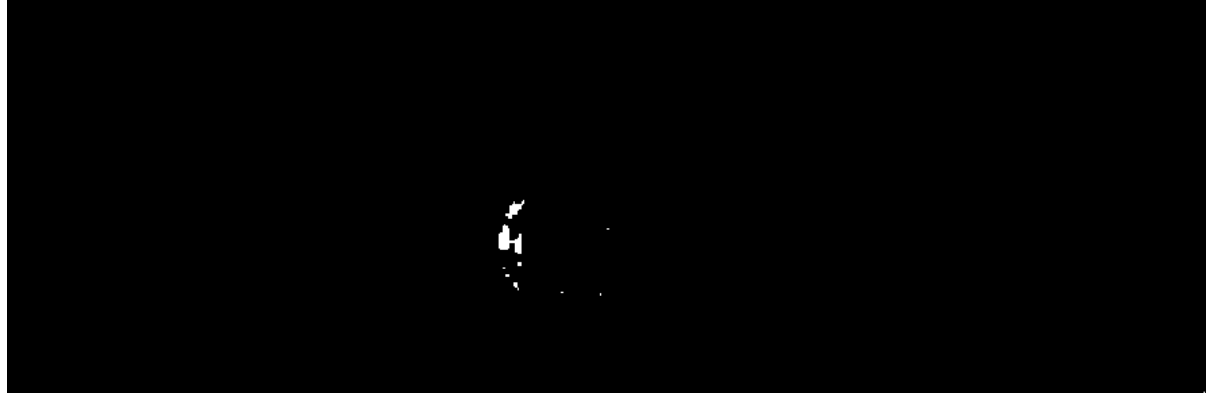

Fig. 4. BGS approach comparison of a case of heavily occlusion

classified as foreground by these pixel-based BGS approaches. Since the MBGS system operates by inserting a model as foreground, this problem is effectively resolved. Figure 4 shows the same scenario, in a frame where the pedestrian is heavily occluded. The occlusion causes the pedestrian to more or less disappear with pixel based approaches. This happens because the occlusion divides the pedestrian contour into separate smaller parts, which are then removed by the subsequently applied morphological image filters (see for details [3]). The scenario presented in figure 5 shows two pedestrians walking towards each other, thereby crossing behind lamp posts and a statue. When processing this scenario, a combination of image filtering and the background variation, renders the silhouettes of the pixel-based approaches unidentifiable. Also both pixel-based approaches severely distorts the contours of the pedestrians. By only inspecting the pixel-based results, it is hard to tell that the foreground objects are actually pedestrians.

A further example can be seen in Fig. 6. A pedestrian is passed by a car. Still, the MBGS system is able to compute a senseful output due to its model knowledge while the pixel-based BGS techniques fail.

In a quantitative evaluation we have investigated the correctness of the particle method in matching the correct contour. In particular, the experiments verify whether the contour selection strategy of applying a Markov transition 


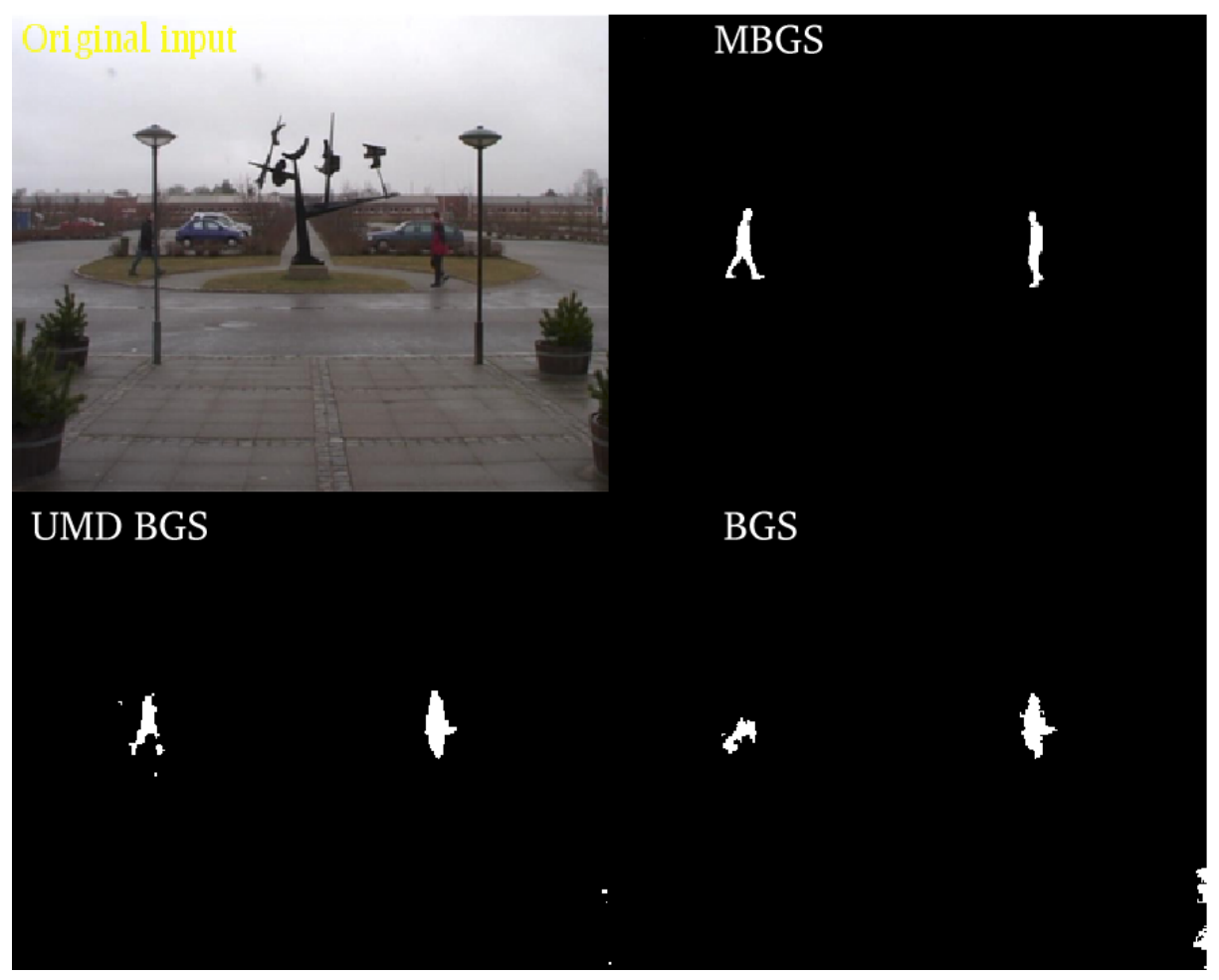

Fig. 5. BGS approach comparison in a situation of low contrast

matrix to choose between silhouette clusters, is suitable. When the MBGS is started, the particles are evenly distributed and the system needed usually 2050 frames to find a sufficiently good approximation of the true density. Then, the selected contour is rather random. After 50 frames, the contour with the maximum likelihood is the correct one in $\approx 98 \%$ of the cases. In $\approx 20 \%$ of the cases the ML contour was incorrect when e.g. a bush was largely occluding the legs. However, recovery time was within 5 frames. In case of partial occlusion of the body through, e.g. small trees, reliability degraded between $1 \%$ (slight occlusion) to $10 \%$ (considerable occlusion), The contour was incorrect in $\approx 59 \%$ of the cases where the individual was largely occluded, e.g. by a big car or bus. In the videos the individual was in average $70 \mathrm{px}$. high. Reliability increased considerably with more pixels on target.

The system has been tested on a $2 \mathrm{GHz}$ Pentium under Linux. In videos of size $320 \times 240$ pixels with only a single person to track, the system runs, with 350 particles, with $\approx 50 \mathrm{~ms} /$ frame: $\approx 25 \mathrm{~ms} /$ frame were used by the classical $\mathrm{BGS}, \approx 25 \mathrm{~ms} /$ frame were used by the matching. 


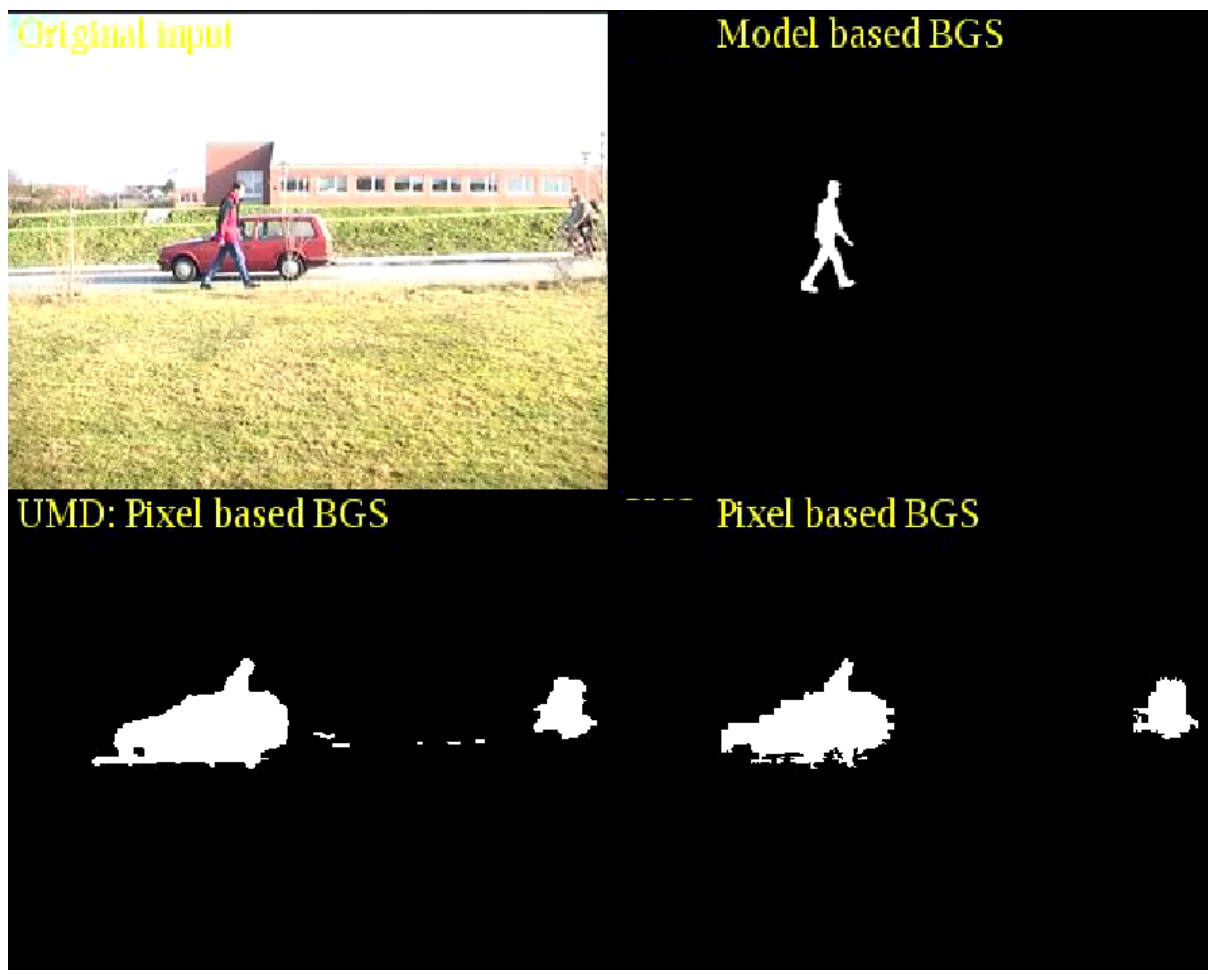

Fig. 6. This figure shows the output of the MGBS when a pedestrian is passed by a car. Compare the to right image (MBGS) with the pixel-based BGS outputs at the bottom

\section{Conclusion}

The presented model-based background subtraction system combines the classical background subtraction with model knowledge of foreground objects. The application of model knowledge is not applied on a binary BGS image but on the "likelihood image", i.e. an image where each pixel value represents a confidence of belonging either to the foreground or background. This approach considerably increases robustness as these likelihoods can also be understood as uncertainties which is exploited for the tracking and silhouette selection process. Also, the propagation of densities prevents the need of selecting thresholds (e.g. for binarization of the image $P$ ) or of maximization. Thresholds are only used for visualization purposes and otherwise for the detection of a new human in the field of view.

In the above application we have chosen silhouettes of humans, but we belive that this choice is without limit of generality since even different object types fit into the tree structure. 
The presented experiments were carried out with only a single individual in the database. We have experimented also with different individuals (and thus varying contours), but the output was instable w.f.t. the choice if the individual. This is under further investigation and the use of our approach for gait recognition is future research.

\section{References}

[1] Dorin Comaniciu, Visvanathan Ramesh, and Peter Meer. Real-time tracking of non-rigid objects using mean shift. In Proc. IEEE Conf. on Computer Vision and Pattern Recognition, volume 2, pages 142-149, Hilton Head Island, SC, June 13-15, 2000.

[2] A. Doucet, S. Godsill, and C. Andrieu. On sequential monte carlo sampling methods for bayesian filtering. Statistics and Computing, 10:197-209, 2000.

[3] A. Elgammal and L. Davis. Probabilistic framework for segmenting people under occlusion. In ICCV, ICCV01, 2001.

[4] D. Gavrila and V. Philomin. Real-time object detection for "smart" vehicles. In Proc. Int. Conf. on Computer Vision, pages 87-93, Korfu, Greece, 1999.

[5] I. Haritaoglu, D. Harwood, and L. Davis. W4s: A real-time system for detection and tracking people in 2.5 D. In Proc. European Conf. on Computer Vision, Freiburg, Germany, June 1-5, 1998.

[6] T. Horprasert, D. Harwood, and L.S. Davis. A statistical approach for real-time robust background subtraction and shadow detection. In Proceedings of IEEE ICCV'g9 FRAME-RATE Workshop, 1999.

[7] M. Isard and A. Blake. Condensation - conditional density propagation for visual tracking. Int. J. of Computer Vision, 1998.

[8] M. Isard and A. Blake. Condensation - conditional density propagation for visual tracking. Int. J. of Computer Vision, 29:5-28, 1998.

[9] Yuri A. Ivanov, Aaron F. Bobick, and John Liu. Fast lighting independent background subtraction. Int. J. of Computer Vision, 37(2):199-207, 2000.

[10] A. Kale, A. Sundaresan, A.N. Rjagopalan, N. Cuntoor, A.R. Chowdhury, V. Krüger, and R. Chellappa. Identification of humans using gait. IEEE Trans. Image Processing, 9:1163-1173, 2004.

[11] G. Kitagawa. Monta carlo filter and smoother for non-gaussian nonlinear state space models. J. Computational and Graphical Statistics, 5:1-25, 1996.

[12] V. Krueger and S. Zhou. Exemplar-based face recognition from video. In Proc. European Conf. on Computer Vision, Copenhagen, Denmark, June 27-31, 2002.

[13] J.S. Liu and R. Chen. Sequential monte carlo for dynamic systems. Journal of the American Statistical Association, 93:1031-1041, 1998.

[14] K. Toyama and A. Blake. Probabilistic tracking in a metric space. In Proc. Int. Conf. on Computer Vision, volume 2, pages 50-59, Vancouver, Canada, 9-12 July, 2001 . 